\title{
Decentralized Data Flows in Algebraic Service Compositions for the Scalability of IoT Systems
}

DOI:

10.1109/WF-IoT.2019.8767238

\section{Document Version}

Accepted author manuscript

Link to publication record in Manchester Research Explorer

\section{Citation for published version (APA):}

Arellanes Molina, D., \& Lau, K-K. (2019). Decentralized Data Flows in Algebraic Service Compositions for the Scalability of loT Systems. In IEEE 5th World Forum on Internet of Things https://doi.org/10.1109/WFIoT.2019.8767238

\section{Published in:}

IEEE 5th World Forum on Internet of Things

\section{Citing this paper}

Please note that where the full-text provided on Manchester Research Explorer is the Author Accepted Manuscript or Proof version this may differ from the final Published version. If citing, it is advised that you check and use the publisher's definitive version.

\section{General rights}

Copyright and moral rights for the publications made accessible in the Research Explorer are retained by the authors and/or other copyright owners and it is a condition of accessing publications that users recognise and abide by the legal requirements associated with these rights.

\section{Takedown policy}

If you believe that this document breaches copyright please refer to the University of Manchester's Takedown Procedures [http://man.ac.uk/04Y6Bo] or contact uml.scholarlycommunications@manchester.ac.uk providing relevant details, so we can investigate your claim.

\section{OPEN ACCESS}




\title{
Decentralized Data Flows in Algebraic Service Compositions for the Scalability of IoT Systems
}

\author{
Damian Arellanes and Kung-Kiu Lau \\ School of Computer Science \\ The University of Manchester \\ Manchester M13 9PL, United Kingdom \\ \{damian.arellanesmolina, kung-kiu.lau\}@manchester.ac.uk
}

\begin{abstract}
With the advent of the Internet of Things, scalability becomes a significant concern due to the huge amount of data involved in IoT systems. A centralized data exchange is not desirable as it leads to a single performance bottleneck. Although a distributed exchange removes the central bottleneck, it has network performance issues as data passes among multiple coordinators. A decentralized data flow exchange is the only solution that fully enables the realization of efficient IoT systems as there is no single performance bottleneck and the network overhead is minimized. In this paper, we present an approach that leverages the algebraic semantics of DX-MAN for realizing decentralized data flows in IoT systems. As data flows are not mixed with control flows in algebraic service compositions, we developed an algorithm that smoothly analyzes data dependencies for the generation of a direct relationship between data consumers and data producers. The result prevents passing data alongside control among multiple coordinators because data is only read and written on a data space. We validate our approach using the Blockchain as the data space and conducted experiments to evaluate the scalability of our approach. Our results show that our approach scales well with the size of IoT systems.

Index Terms-Internet of Things, decentralized data flows, Blockchain, DX-MAN, exogenous connectors, scalability, separation between control and data, algebraic service composition
\end{abstract}

\section{INTRODUCTION}

The Internet of Things (IoT) envisions a world where everything will be interconnected through distributed services. As new challenges are forthcoming, this paradigm requires a shift in our way of building software systems. With the rapid advancement in hardware, the number of connected things is considerably increasing to the extent that scalability becomes a significant concern due to the huge amount of data involved in IoT systems. Thus, IoT services shall exchange data over the Internet using efficient approaches.

Although a centralized data exchange approach has been successful in enterprise systems, it will easily cause a bottleneck in IoT systems which potentially generate huge amount of data continuously. To avoid the bottleneck, a distributed approach can be used to distribute the load of data over multiple coordinators. However, this would introduce unnecessary network overhead as data is passed among many loci of control.

A decentralized data exchange approach is the most efficient solution to tackle the imminent scale of IoT systems, as it achieves better response time and throughput by minimizing network hops [1], [2], [3], [4], [5]. However, exchanging data among loosely-coupled IoT services is challenging, specially in resource-constrained environments where things have poor network connection and low disk space.

Moreover, constructing data dependency graphs is not trivial when control flow and data flow are tightly coupled. The separation of such concerns would allow a separate reasoning, monitoring, maintenance and evolution of both control and data [6]. Consequently, an efficient data exchange approach can be realized without considering control flow. Thus, the number of messages transmitted over the Internet can be reduced considerably.

This paper proposes an approach that leverages the algebraic semantics of DX-MAN [7], [8] for the realization of decentralized data flows in IoT systems. The algebraic semantics of DX-MAN allows a well-defined structure of data flows which are smoothly analyzed by an algorithm, in order to form a direct relationship between data consumers and data producers. For this analysis, the algorithm particularly takes advantage of the fact that DX-MAN separates control flow and data flow.

The rest of the paper is organized as follows. Sect. II introduces the composition semantics of the DX-MAN model. Sect. III describes its data flow dimension. Sect. IV presents the algorithm that analyzes data flows. Sect. V presents the implementation of our approach. Sect. VI outlines a quantitative evaluation of our approach. Finally, we present the related work in Sect. VII and the conclusions in Sect. VIII.

\section{DX-MAN MODEL}

DX-MAN is an algebraic model for IoT systems where services and exogenous connectors are first-class entities. An exogenous connector is a variability operator that defines multiple workflows with explicit control flow, while a DXMAN service is a distributed software unit that exposes a set of operations through a well-defined interface.

An atomic service provides a set of operations and it is formed by connecting an invocation connector with a computation unit. A computation unit represents an actual service implementation (e.g., a RESTful Microservice or a WS-* service) and it is not allowed to call other computation units. The red arrows in Fig. 1(a) show that, as a consequence of the algebraic semantics, the interface of an atomic service has all the operations in the computation unit. An invocation 
connector defines the most primitive workflow which is the invocation of one operation in the computation unit.

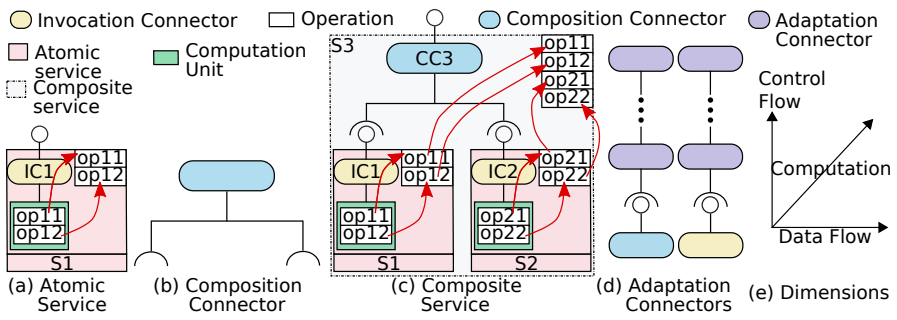

Fig. 1. DX-MAN Model.

Our notion of algebraic service composition is inspired by algebra where functions are hierarchically composed into a new function of the same type. The resulting function can be further composed with other functions so as to yield a more complex one. Algebraic service composition is the operation by which a composition connector is used as an operator to compose multiple services, resulting in a (hierarchical) composite service whose interface has all sub-service operations. Thus, a top-level composite will always contains the operations of all atomic services. Fig. 1(c) illustrates this concept. In particular, there are composition connectors for sequencing, branching and parallelism. A sequencer connector enables infinite workflows for the sequential invocation of sub-service operations. A selector connector defines $2^{n}$ branching workflows and chooses the sub-service operations to invoke, such that $n$ is the number of operations in the composite service interface. A parallel connector defines $2^{n}$ parallel workflows and executes subservice operations in parallel according to user-defined tasks.

Fig. 1(d) shows that an adapter can be connected with only one exogenous connector. A looping adapter iterates over a sub-workflow while a condition holds true, and a guard adapter invokes a sub-workflow whenever a condition holds true. There are also adapters for sequencing, branching and parallelism over the operations of an individual atomic service.

Fig. 1(e) shows that control, data and computation are orthogonal dimensions in DX-MAN. Exogenous connectors enable the separation between control flow and computation, since they decouple service implementations from the (hierarchical) composition structure. Unlike existing composition approaches, data flow never follows control flow as exogenous connectors only pass control to coordinate workflow executions. For further details about the control flow dimension, we refer the read to our previous papers [7], [8].

\section{DATA CONNECTORS}

A DX-MAN operation is a set of input parameters and output parameters. An input parameter defines the required data to perform a computation, while an output parameter is the resulting data from a specific computation. Although exogenous connectors do not provide any operation (because they do not perform any computation), some of them require data. In particular, selector connectors, looping adapters and guard adapters require input values to evaluate boolean conditions. Connectors do not have any parameters by default since designers define the parameters they require when choosing a workflow. Workflow selection is out of the scope of this paper, but we refer the read to our previous paper on workflow variability [8].

In addition to the operations created on algebraic composition, custom operations can be defined in composite services. This is particularly useful when designers want to hide the operations created during algebraic composition or when designers want to create a unified interface for a composite service.

A data connector defines explicit data flow by connecting a source parameter with a destination parameter. Fig. 2 shows that an algebraic data connector is automatically created during composition and it is available for all the workflows defined by a composite. In particular, an algebraic data connector connects two parameters vertically, i.e., in a bottom-up way for outputs or in a top-down fashion for inputs. The top-down approach connects a parameter of a composite service operation to a parameter of a sub-service operation, whilst the bottom-up approach means the other way round. Fig. 3 shows the data connection rules, where we can see that the algebraic data connectors are defined in four different ways.

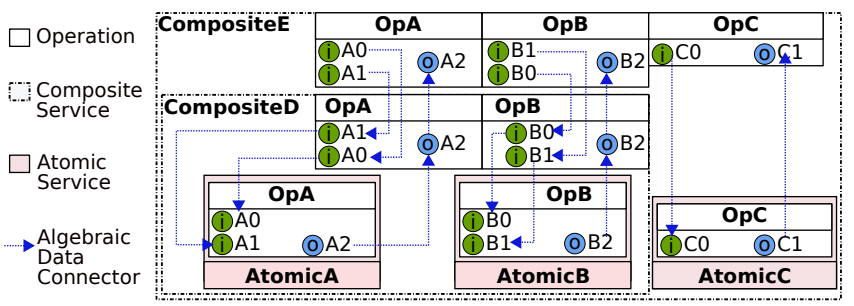

Fig. 2. Algebraic data channels.

A custom data connector is manually created by a designer for only one workflow. Custom data connectors connect two parameters either vertically or horizontally. An horizontal approach connects the parameters of two sub-service operations, or an operation parameter with an exogenous input. A quick glance at Fig. 3 reveals that a designer is allowed to connect parameters in 16 different ways.

A designer uses custom data connectors to define data flows for a particular workflow. Currently, DX-MAN supports the most common patterns: sequencing and map-reduce. For the sequencing pattern, the parameters of two different operations are horizontally connected. Fig. 4 shows an example of this pattern, where operation $O p B$ requires data from operation $O p A$. In particular, a custom data connector links the output $A O$ with the input $B 0$, while another custom data connector connects the output $A l$ with the input $B l$. To improve readability, we ignore algebraic data connectors.

A data processor is particularly useful when data preprocessing needs to be done before executing an operation. It waits until all input values have been received, then performs some computation and returns transformed data in the form of outputs. A mapper executes a user-defined function on each input value received. A reducer takes the result from a mapper and executes a user-defined reduce function on inputs. A reducer can also be used in isolation to perform straightforward computation such as combining data into a list. Fig. 5 shows 


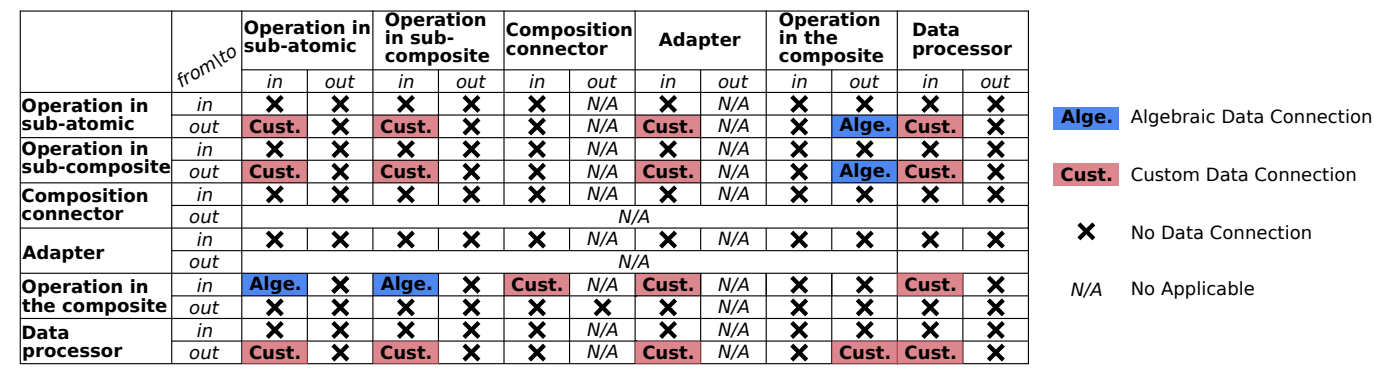

Fig. 3. Data connection rules.

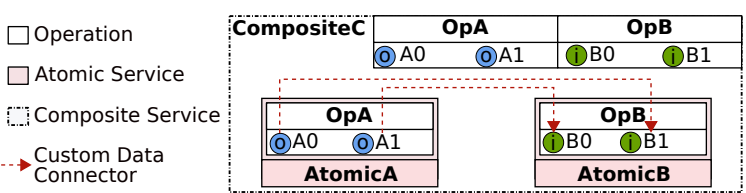

Fig. 4. An example of two sequential data flows.

an example of the map-reduce pattern, where operation $o p B$ requires the pre-processing of data generated by operation $o p A$. In particular, two custom data connectors link the input $A O$ and the output $A l$ with the inputs of the mapper. The output of the mapper is connected to the input of the reducer and, similarly, the output of the reducer is connected to the input $B O$. Please note that $A O$ can only be connected from the composite service operation, according to the rules shown in Fig. 3.

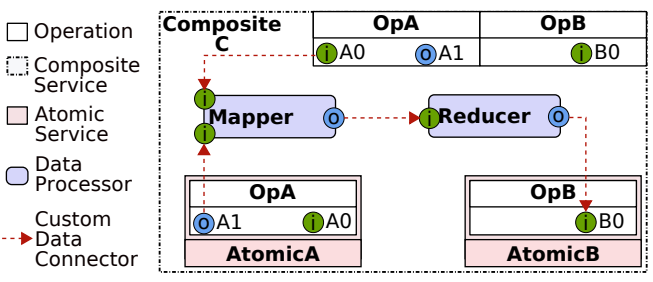

Fig. 5. An example of the map-reduce pattern.

In some workflows, algebraic data connectors may not be useful. For that reason, such connectors can be removed manually at the discretion of the designer. For example, in Fig. 5 all algebraic connectors were removed because data is only needed for the realization of the map-reduce pattern. At quick glance at Fig. 4 reveals that composite services encapsulate data flows to ensure reusability. Thus, composite services are black boxes who are not aware of data flows of other composites.

\section{Analysis of Data Connectors}

Algebraic service composition and the separation of concerns are key enablers for the realization of decentralized data flows. The separation between control and data allows a separate reasoning of these dimensions. In particular, exogenous connectors provide a hierarchical control flow structure that is completely separated from the data flow structure enabled by data connectors. The data connections in a composite service form a well-structure data dependency graph that is analyzed at deployment-time by means of the Algorithm 1. To understand this algorithm, it is necessary to underline some formal definitions.

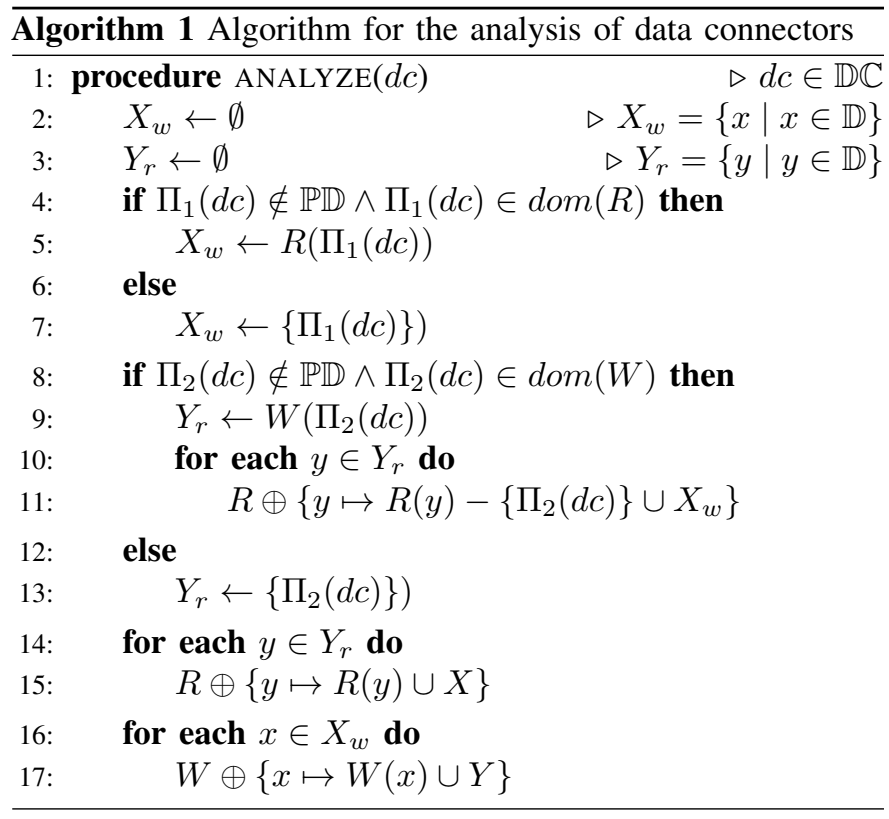

Let $\mathbb{D}$ be the data type, $\mathbb{P D}$ the type of processor parameters, $\mathbb{O D}$ the type of operation parameters and $\mathbb{C D}$ the type of exogenous connector inputs, such that $\mathbb{P D}, \mathbb{O D}, \mathbb{C D} \subseteq \mathbb{D}$. A data connector is then a tuple of type $\mathbb{D} \mathbb{C}: \mathbb{D} \times \mathbb{D}$ that connects a source $\in \mathbb{D}$ parameter with an origin $\in \mathbb{D}$ parameter.

Reader parameters are the entities that directly consume data produced by writer parameters. $I_{r}$ is the set of inputs that read data during a workflow execution, namely the inputs of atomic service operations, the inputs of exogenous connectors and the inputs of data processors. $O_{r}$ is the set of operation outputs in the top-level composite, useful for reading data resulting from a workflow execution. The set $I_{w}$ represents the required data for a workflow execution, which are the inputs of operations in the top-level composite. $O_{w}$ is the set of outputs that write data during a workflow execution, namely the outputs of atomic service operations and the outputs of data processors.

Basically, the Algorithm 1 analyzes data connectors for all composite services, in order to create a relationship between reader parameter and writer parameters, while ignoring those parameters who do not need to manipulate data. It receives a data connector $d c \in \mathbb{D C}$ as an input, and uses $R=I_{r} \cup O_{r} \mapsto$ $\left\{w \mid w \subset I_{w} \cup O_{w}\right\}$ for mapping a reader parameter to a set of writer parameters and $W=I_{w} \cup O_{w} \mapsto\left\{r \mid r \subset I_{r} \cup O_{r}\right\}$ for mapping a writer parameter to a set of reader parameters. 
The Algorithm 1 creates two empty sets $X_{w}$ and $Y_{r}$, in order to analyze the endpoints of a data connector $d c \in D C . X_{w}$ is the set of parameters connected to the source parameter $\Pi_{1}(d c)$ if $f \Pi_{1}(d c)$ is not a data processor parameter and $\Pi_{1}(d c)$ has incoming data connectors; otherwise, $X_{w}$ only contains $\Pi_{1}(d c)$. Similarly, if the destination parameter $\Pi_{2}(d c)$ is not a data processor parameter and $\Pi_{2}(d c)$ has outgoing data connectors, then $Y_{r}$ is the set of parameters connected from $\Pi_{2}(d c)$ and $X_{w}$ (without $\Pi_{2}(d c)$ ) is added into the writers of each element $y \in Y_{r}$; otherwise, $Y_{r}$ only contains $\Pi_{2}(d c)$. Finally $X_{w}$ is added into the writers of each element $Y \in Y_{r}$, while the set $Y_{r}$ is added into the readers of each element $x \in X_{w}$. The result of the algorithm is a mapping of reader parameters to writer parameters.

\section{IMPLEMENTATION}

We implemented our approach on top of the DX-MAN Platform [9], and we used the Blockchain as the underlying data space for persisting parameter values while leveraging the capabilities provided by these decentralized platforms, such as performance, security and auditability. Furthermore, the Blockchain ensures that every service is the owner of its own data, while data provenance is provided to discover data flows (i.e., how data is moved between services) or to find out how parameters change over time. In particular, we defined three smart contracts using Hyperledger Composer 0.20.0 for executing transactions on Hyperledger Fabric 1.2. We do not show the source code due to space constraints, but it is available at .

The DX-MAN platform provides an API to support the three phases of a DX-MAN system lifecycle: design-time, deployment-time and run-time. Composite service templates only contain algebraic data connectors, as they represent a general design with multiple workflows. Using API constructs, a designer chooses a workflow and defines custom data connectors (and perhaps data processors) for every composite service involved. Data processor functions are defined by designers using API constructs.

The Algorithm 1 analyzes the data connectors defined at design-time, in order to construct the readers map at deployment-time. In particular, the map is a Java HashMap where the keys are reader parameter UUIDs and the values are lists of writer parameter UUIDs. After getting the map for a given workflow, reader parameters (with their respective list of writers) are stored as assets in the Blockchain by means of the transaction CreateParameters.

At run-time, exogenous connectors pass control using CoAP messages. In particular, an invocation connector performs five steps to invoke an operation, as shown in Fig. 6. Although the rest of exogenous connectors behave similarly, they only perform the first two steps. First, the invocation connector uses the transaction readParameters to read all input values from the Blockchain. For a given input, the Blockchain reads values directly from the writers list. As there might be multiple writer parameters, this transaction returns a list of the most recent input values that were updated during the workflow execution. Hence, a timestamp is set whenever a parameter value is updated. readParameters returns an exception if there are no input values. Output values are written onto the data space as soon as they are available, even before control reaches data consumers. Thus, having concurrent connectors (e.g., a parallel connector) may lead to synchronization issues during workflow execution. To solve this, control flow blocks in the invocation connector until all input values are read.

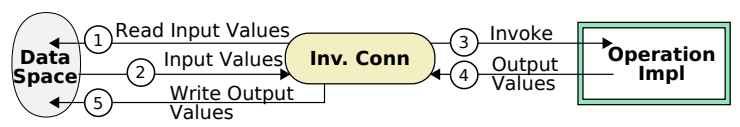

Fig. 6. Steps for the invocation of an operation implementation.

Once all inputs are ready, the invocation connector invokes the implementation of an operation by passing the respective input values. Then, the operation performs some computation and returns the result in the form of outputs. Finally, the invocation connector writes the output values onto the Blockchain using the transaction updateParameters.

An UpdateParameterEvent is published whenever a new parameter value has been updated. During deployment, the platform automatically subscribes data processor instances to the events produced by the respective writer parameters. Thus, a data processor instance waits until it receives all events, before performing its respective designer-defined computation. Although our current implementation supports only mappers and reducers, more data processors can be introduced using the semantics of a data processor presented in Sect. III, e.g., we can add a shuffler to sort data by key.

Our approach enables transparent data exchange as data routing is embodied in the Blockchain. Thus, reader parameters are not aware where the data comes from, and writer parameters do not know who reads the data they produce. Furthermore, the map generated by the Algorithm 1 avoids the inefficient approach of passing values through data connectors during workflow execution. Thus, exogenous connectors and data processors read data directly from parameters who only write values onto the Blockchain. Undoubtedly, this enables a transparent decentralized data exchange.

\section{Evaluation}

In this section, we present a comparative evaluation between distributed data flows and decentralized data flows for a DXMAN composition. In the former approach, data is passed over the network through data connectors, whereas the second approach is our solution. Our evaluation intends to answer two major research questions: (A) Does the approach scale with the number of data connectors? and (B) Under which conditions is decentralized data exchange beneficial?

As a DX-MAN composition has a multi-level hierarchical structure, an algebraic data connector passes a data value vertically in a bottom-up way (for inputs) or in a top-down fashion (for outputs) while a custom data connector passes values horizontally or vertically. For our evaluation, we only consider vertical routing through algebraic data connectors.

$M_{p}=\left\{\lambda_{j} \mid \lambda_{j} \in \mathbb{R}\right\}$ is the set of network message costs for vertically routing the value of a parameter $p$, where $\lambda_{j}$ is the 
cost of passing that value through an algebraic data connector $j$. Likewise, $\Gamma_{p}$ and $\omega_{p}$ are the costs of reading and writing the value on the data space, respectively.

Equations 1 and 2 calculate the total message cost of routing a value with a distributed approach. In particular, equation 1 is used for input values, whilst equation 2 is used for output values. As the decentralized approach does not pass values through data connectors, the total message cost of routing the value of $p$ is $\Gamma_{p}$ for inputs, and $\omega_{p}$ for outputs.

$$
\begin{gathered}
\Gamma_{p}+\sum_{j=0}^{\left|M_{p}\right|-1} \lambda_{j} \\
\omega_{p}+\sum_{j=0}^{\left|M_{p}\right|-1} \lambda_{j}
\end{gathered}
$$

Fig. 7 depicts the DX-MAN composition that we consider for our evaluation, which has three levels, three atomic services and two composite services. The composites ServiceD and ServiceE have three and five data connectors, respectively. Fig. 7 shows that a data connector has a $\lambda_{j \in[0,7]}$ cost of passing a value over the network. Then, the vertical routing sets for the parameters are $M_{A 0}=\left\{\lambda_{3}\right\}, M_{A 1}=\left\{\lambda_{4}\right\}, M_{B 0}=\left\{\lambda_{0}, \lambda_{5}\right\}$, $M_{B 1}=\left\{\lambda_{1}, \lambda_{6}\right\}$ and $M_{C 0}=\left\{\lambda_{2}, \lambda_{7}\right\}$.

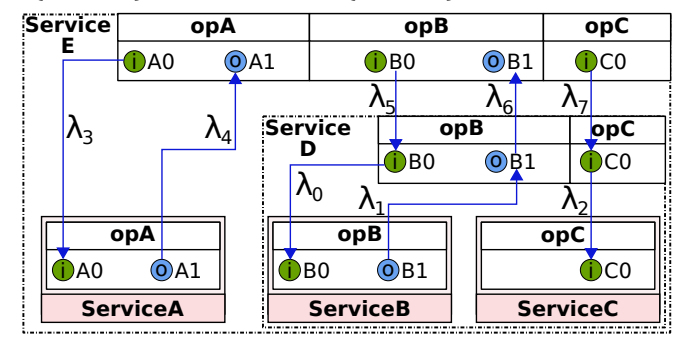

Fig. 7. DX-MAN composition for the evaluation of our approach.

For clarity, we assume that the DX-MAN composition interacts with an external application through a shared data space. So, we can ignore the cost of passing data between the application and the composition. The costs of reading the inputs $A O, B O$ and $C O$ are $\Gamma_{A 0}, \Gamma_{B 0}$ and $\Gamma_{C 0}$, respectively, and the costs of writing the outputs $A l$ and $B 1$ are $\omega_{A 1}$ and $\omega_{B 1}$, respectively.

Suppose that a specific workflow requires the invocation of the operations $o p A$ and $o p C$. Using a distributed approach would require passing and reading values for two inputs, and returning and writing one output value. Therefore, according to equations 1 and 2, the total message cost would be $\lambda_{3}+\lambda_{4}+$ $\lambda_{2}+\lambda_{7}+\Gamma_{A 0}+\omega_{A 1}+\Gamma_{C 0}$. Remarkably, the total message cost using the decentralized approach would be $\Gamma_{A 0}+\omega_{A 1}+\Gamma_{C 0}$.

\section{A. RQ1: Does the approach scale with the number of data connectors?}

We conducted an experiment that dynamically increases the number of data connectors of the DX-MAN composition depicted in Fig. 7. The experiment is carried out in 100000 steps with $\Gamma_{A 0}=\omega_{A 1}=\Gamma_{B 0}=\omega_{B 1}=\Gamma_{C 0}=1$.

For each step of the experiment, we add a new parameter in a random atomic operation. As a consequence of algebraic composition, another parameter is added in the respective composite operation and a data connector links these parameters.

In this experiment, we particularly compare the cost of the distributed approach vs. the cost of the decentralized approach. Rather than computing the costs for the invocation of specific operations, we compute the total costs for the DX-MAN composition using $\Gamma_{A 0}+\omega_{A 1}+\Gamma_{B 0}+\omega_{B 1}+\Gamma_{C 0}+\sum_{j=0}^{7} \lambda_{j}$. Fig. 8 shows that the costs grow linearly with the number of data connectors, and that the decentralized approach outperforms its counterpart by reducing costs by a factor of 2.67 in average.

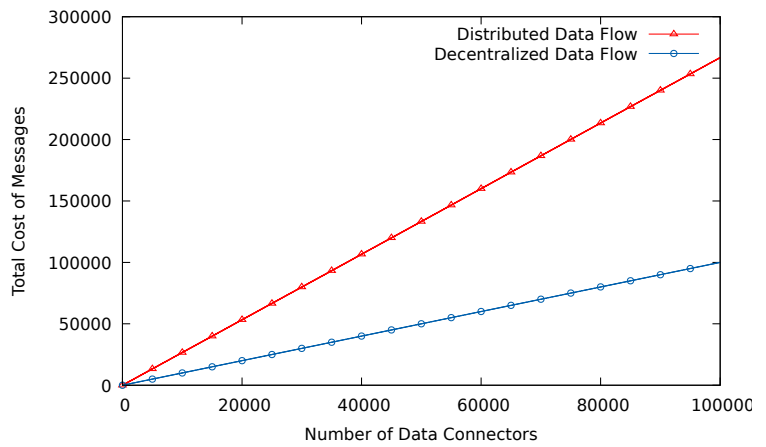

Fig. 8. Impact of increasing the number of data connectors in a DX-MAN composition.

B. RQ2: Under which conditions is decentralized data exchange beneficial?

We conducted an experiment of 100000 steps to see the benefit of the decentralized approach as the number of levels of the composition increases. We particularly consider the total costs for the input $A O$ and we assume that $\Gamma_{A 0}=1$. At each step, the number of levels is increased by 1 and $\sum_{j=0}^{\left|M_{A 0}\right|-1} \lambda_{j}$ by 0.0004 . Thus, increasing the sum of vertical costs means $\left|M_{A 0}\right|-1$

that $\frac{\sum_{j=0} \lambda_{j}}{\left|M_{A 0}\right|}=1$ and increasing the number of levels by 1 means that $\left|M_{A 0}\right|$ is also increased by 1 . The improvement rate of the decentralized data exchange is $1-\frac{\Gamma_{A 0}}{\left(\Gamma_{A 0}+\sum_{j=0}^{\left|M_{A 0}\right|-1} \lambda_{j}\right)}$

Fig. 9 shows the results of this experiment, where it is clear that the benefit of the decentralized approach becomes more evident as the number of levels of the composition increases. This is because the number of data connectors increases with the number of levels and so the cost of the distributed approach. The only way a distributed approach would outperform the decentralized one is when the cost of performing operations on the data space is more expensive than the total cost of passing values vertically. In particular, for our experiment the DX-MAN composition gets a benefit only if $\Gamma_{A 0}<\sum_{j=0}^{\left|M_{A 0}\right|-1} \lambda_{j}$.

\section{RELATED WORK}

To the best of our knowledge, there are no solutions to enable decentralized data flows in IoT systems. In this section we present SOA-based solutions as they are applicable to IoT. 


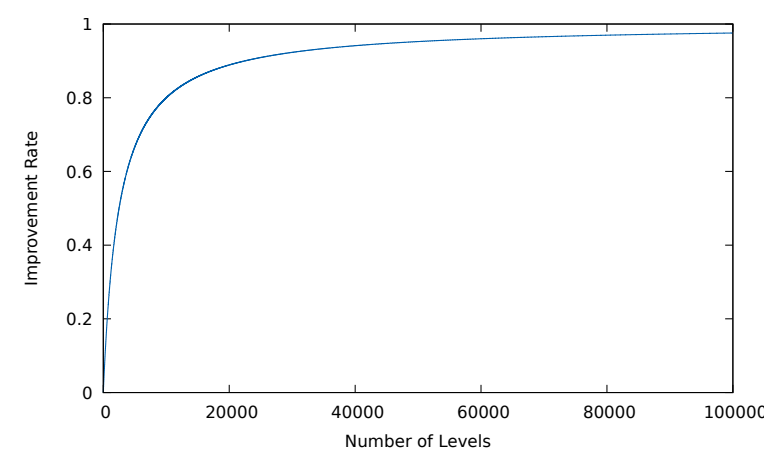

Fig. 9. Impact of increasing the number of levels in a DX-MAN composition.

We classified our findings into three categories, depending on the composition semantics the approaches are built on: orchestration (with central control flows and decentralized data flows), decentralized orchestration, data flows and choreographies.

Approaches belonging to the first category [10], [1] partially separate data from control so as to enable $\mathrm{P} 2 \mathrm{P}$ data exchanges. To do so, an orchestrator coordinates the exchanges by passing data references alongside control. Thus, extra network traffic is introduced as data references (and acknowledge messages) are transferred over the network. These approaches are typically based on proxies that keep data, thus representing an issue for things with low disk space. By contrast, DX-MAN does not require any coordinator for the data exchange, and exogenous connectors do not store data. Besides, exogenous connectors do not exchange references, thanks to the separation of concerns.

Only few approaches discuss data decentralization using the semantics of decentralized orchestration. [11] stores data and control in distributed tuple spaces which may become a bottleneck in IoT environments that continuously generate huge amount of data. [3] solves that issue by storing references instead of values. However, references are needed because data is mixed with control. Moreover, [3] requires the maintenance of tuple spaces for passing references and databases for storing data. DX-MAN only reads and writes onto the data space.

Although distributed data flows [12] allocate flows over different things, there is a master engine that coordinates data exchange for slave engines. Hence, this approach introduces extra network hops as data is passed among multiple engines. Although Service Invocation Triggers [2] exchange data directly, they rely on workflows that do contain loops and conditionals. This limitation arises from the fact that it is not trivial to analyze data dependencies when control is mixed with data.

A choreography describes interactions among participants using decentralized message exchanges (a.k.a. conversations). Workflow participants [13], pass data among multiple engines leading to network degradation. Although services may exchange data through direct message passing, they are not reusable because data and control are mixed [6]. [4] uses peers to exchange data and invoke services, thus separating control and computation. However, peers pass data alongside control according to predefined conversations, leading to the issues discussed in [5]. Although [14] proposes the separation between control and data for choreographies, it uses a middleware which may potentially become a central bottleneck.

\section{CONCLUSIONS}

In this paper, we presented an approach on top of DXMAN to enable decentralized data flows in IoT systems. At design-time, the algebraic semantics of DX-MAN enables a well-defined structure of data connections. As data connections are not mixed with control flow structures, then an algorithm smoothly analyzes data connections at deployment-time. The result is a mapping between reader parameters and writer parameters, which prevents passing values through data connectors. In our current implementation, the Blockchain embodies this mapping to manage data values at run-time.

DX-MAN is currently the only service model that provides the separation between data flow, control flow and computation; thus, allowing a separate reasoning, monitoring, maintenance and evolution of these concerns. In particular, separating data flow from control flow prevents passing data alongside control among exogenous connectors, and enables the use of different technologies to handle data flows and control flows separately.

Our experiments confirm that our approach scales well with the number of data connectors and the number of levels of a DX-MAN composition. They also suggest that our approach provides the best performance when the cost of performing operations on the data space is less than the cost of passing data over the network. Thus, our approach is extremely beneficial for IoT systems consisting of plenty of services.

\section{REFERENCES}

[1] A. Barker et al., "Reducing Data Transfer in Service-Oriented Architectures: The Circulate Approach," IEEE Trans. Serv. Comput., vol. 5 , no. 3, pp. 437-449, 2012.

[2] W. Binder et al., "Service invocation triggers: a lightweight routing infrastructure for decentralised workflow orchestration," Int. J. High Perf. Comp. and Net., vol. 6, no. 1, pp. 81-90, 2009.

[3] M. Sonntag et al., "Process space-based scientific workflow enactment," Int. J. Business Proc. Integr. and Man., vol. 5, no. 1, pp. 32-44, 2010.

[4] A. Barker et al., "Choreographing Web Services," IEEE Trans. Serv. Comput., vol. 2, no. 2, pp. 152-166, 2009.

[5] M. Hahn et al., "Data-Aware Service Choreographies Through Transparent Data Exchange," in Web Eng., ser. Lect. Notes Comp. Sci. Springer Int. Pub., 2016, pp. 357-364.

[6] D. Arellanes and K. Lau, "Analysis and Classification of Service Interactions for the Scalability of the Internet of Things," in IEEE ICIOT, 2018, pp. 80-87.

[7] D. Arellanes and K.-K. Lau, "Exogenous Connectors for Hierarchical Service Composition," in IEEE SOCA, 2017, pp. 125-132.

[8] —_ "Algebraic Service Composition for User-Centric IoT Applications," in ICIOT 2018, ser. Lect. Notes Comp. Sci. Springer Int. Pub., 2018, pp. 56-69.

[9] D. Arellanes and K. Lau, "D-XMAN: A Platform For Total Compositionality in Service-Oriented Architectures," in IEEE SC2, 2017, pp. 283-286.

[10] D. Liu, "Data-flow Distribution in FICAS Service Composition Infrastructure," 2002.

[11] D. Wutke et al., "Model and Infrastructure for Decentralized Workflow Enactment," in Proc. Symp. on Appl. Comp. ACM, 2008, pp. 90-94.

[12] N. K. Giang et al., "Developing IoT applications in the Fog: A Distributed Dataflow approach," in IOT, 2015, pp. 155-162.

[13] G. Decker et al., "BPEL4chor: Extending BPEL for Modeling Choreographies," in IEEE ICWS, 2007, pp. 296-303.

[14] M. Hahn et al., "TraDE - A Transparent Data Exchange Middleware for Service Choreographies," in On the Move to Meaningful Internet Syst., ser. Lect. Notes Comp. Sci. Springer Int. Pub., 2017, pp. 252-270. 